\title{
Influence of left ventricular function on development of systolic anterior motion after mitral valve repair
}

\author{
Susumu Manabe, MD, ${ }^{\mathrm{a}}$ Hitoshi Kasegawa, MD, ${ }^{\mathrm{b}}$ Toshihiro Fukui, MD, ${ }^{\mathrm{b}}$ Minoru Tabata, MD, MPH, ${ }^{\mathrm{b}}$ \\ Tomohiro Shinozaki, MPH, ${ }^{\mathrm{c}}$ Tomoki Shimokawa, MD, ${ }^{\mathrm{a}}$ and Shuichiro Takanashi, MD ${ }^{\mathrm{b}}$
}

Objective: A hyperkinetic heart has been suggested as a risk factor for systolic anterior motion (SAM) after mitral valve repair, but the influence of preoperative left ventricular (LV) function on the development of SAM has not been elucidated.

Methods: Transthoracic echocardiographic data were retrospectively reviewed in 441 patients who underwent mitral valve repair for degenerative mitral regurgitation. Comparisons were made between patients with and without SAM (SAM cases vs noncases).

Results: The incidence of SAM was $6.1 \%$ (27/441). There were no differences in preoperative characteristics and operative procedures between the 2 groups except the prevalence of Barlow disease. The SAM cases exhibited a higher preoperative ejection fraction (EF) (SAM cases, $70.0 \% \pm 7.1 \%$; noncases, $65.1 \% \pm 6.9 \%$; $P<.01)$ and smaller preoperative systolic LV end-systolic dimension (LVDs) $(32.0 \pm 5.4 \mathrm{~mm}$ vs $35.4 \pm 5.7$ $\mathrm{mm} ; P=.02)$ than the noncases. The incidence of SAM was significantly associated with greater preoperative EF $(P<.01$ for trend) and reduced LVDs $(P<.01$ for trend). SAM did not occur in patients with an impaired $(\mathrm{EF}<60 \%$ ) or enlarged (LVDs $>45 \mathrm{~mm}$ ) LV. The incidence of SAM was highest among patients with a small hyperkinetic heart.

Conclusions: The study indicates that the development of SAM after mitral valve repair is associated with preoperative LV function. A small hyperkinetic heart is considered a risk factor for SAM and should be treated with caution. (J Thorac Cardiovasc Surg 2013;146:291-5)

Supplemental material is available online.

Systolic anterior motion (SAM) of mitral leaflets is a common complication that occurs in $8.4 \%$ to $9.8 \%$ of patients who undergo mitral valve repair. ${ }^{1,2}$ In most cases SAM resolves with conservative management, ${ }^{1,2}$ but some patients experience hemodynamic collapse that requires surgical correction. ${ }^{3,4}$ Thus, the possible postoperative emergence of SAM is still a matter of concern and must be managed appropriately by the surgeon who performed the mitral valve operation.

The mechanism of SAM is considered multifactorial. According to Carpentier, Adams, and Filsoufi, ${ }^{5}$ the major risk factors of SAM are excess leaflet tissue and an undersized

\footnotetext{
From the Department of Cardiovascular Surgery, ${ }^{\text {a }}$ Teikyo University Hospital, the

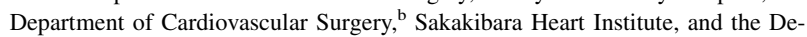
partment of Biostatistics, ${ }^{\mathrm{c}}$ The University of Tokyo, Tokyo, Japan.

Disclosures: Authors have nothing to disclose with regard to commercial support.

Received for publication Feb 27, 2012; revisions received June 11, 2012; accepted for publication July 10, 2012; available ahead of print Aug 10, 2012.

Address for reprints: Susumu Manabe, MD, Department of Cardiovascular Surgery, Teikyo University Hospital, Kaga 2-11-1, Itabashi, Tokyo 183-0003, Japan (E-mail: s-manabe@fb3.so-net.ne.jp).

$0022-5223 / \$ 36.00$

Copyright (C) 2013 by The American Association for Thoracic Surgery http://dx.doi.org/10.1016/j.jtcvs.2012.07.015
}

annular ring, whereas there are several minor risk factors, such as a narrow aorta-mitral angle, abnormal configuration of the anterior leaflet, and a small, hyperkinetic heart. Previous studies have investigated the mechanism of SAM mostly from a morphologic aspect, ${ }^{6-9}$ and there is little known regarding the role of left ventricular (LV) function. Several studies suggested that LV function also has a bearing on the development of SAM, ${ }^{2,4,10}$ but these suggestions were based on the surgeon's impression rather than a systematic analysis using objective data. Hence, the purpose of this study was to investigate the influence of LV function on the development of SAM after mitral valve repair using transthoracic echocardiographic data.

\section{PATIENTS AND METHODS}

\section{Study Design}

This retrospective cohort study investigated the hypothesis that preoperative LV function affects the susceptibility to SAM after mitral valve repair Preoperative echocardiographic indices were compared between patients with and without SAM. The Ethics Committee of Sakakibara Heart Institute approved this study, waived the need for patient consent, and provided approval before the publication of the data.

\section{Patient Population}

Mitral valve repair for degenerative mitral regurgitation (MR) was performed in 503 patients at the Sakakibara Heart Institute between January 2004 and December 2008. The exclusion criterion was concomitant performance of other operative procedures, such as coronary artery bypass grafting $(n=33)$, aortic valve replacement $(n=9)$, left ventriculoplasty $(n=3)$, 


$$
\begin{aligned}
& \text { Abbreviations and Acronyms } \\
& \qquad \begin{array}{l}
\mathrm{CI} \quad=\text { confidence interval } \\
\mathrm{EF}=\text { ejection fraction } \\
\mathrm{LV}=\text { left ventricular (ventricle) } \\
\mathrm{LVDs}=\text { systolic left ventricular dimension } \\
\mathrm{MR}=\text { mitral regurgitation } \\
\mathrm{RR}=\text { risk ratio } \\
\mathrm{SAM}=\text { systolic anterior motion }
\end{array}
\end{aligned}
$$

aortic aneurysm repair $(n=4)$, and repair of an atrial septal defect $(n=4)$. Patients undergoing a concomitant maze procedure or tricuspid valve repair were enrolled in this study. Among the remaining 450 patients, preoperative transthoracic echocardiography was recorded and available for 441 $(98.0 \%)$ patients, who were included in the study. Comparisons were made between patients with and without SAM (SAM cases vs noncases).

\section{Operative Strategy}

The operation was performed by a median sternotomy, and cardiopulmonary bypass was established with aortic and bicaval cannulation. Cold antegrade and retrograde cardioplegia were used for myocardial protection. The mitral valve was exposed through a superior transseptal approach. The standard technique for valve repair was performed according to the anatomic lesions responsible for MR. Chordal replacement with expanded polytetrafluoroethylene was used to repair an anterior mitral leaflet prolapse, and quadrangular resection was used to repair a posterior mitral leaflet prolapse. The sliding technique was used in patients with a posterior leaflet prolapsed by more than $1.5 \mathrm{~cm}$. Annuloplasty was performed in all except 3 study patients. A ring size was chosen according to the area of the anterior leaflet, in line with the widely established techniques of mitral repair in degenerative mitral disease. Residual MR was quantitatively assessed using transesophageal echocardiography. Revision of repair was performed if the MR area was more than $0.1 \mathrm{~cm}^{2}$.

\section{Diagnosis and Management of SAM}

Intraoperative transesophageal echocardiography was performed in all patients undergoing mitral valve repair. SAM was defined as any portion of the anterior mitral valve leaflet protruding into the LV outflow tract. Patients with chordal SAM were excluded.

For patients in whom SAM developed intraoperatively, conservative management was initially performed. This included discontinuing any inotropic drugs, augmenting intravascular volume, and administering $\beta$-blockade as tolerated. When SAM persisted after conservative management, revision of the repair was performed. For most patients with SAM, mitral annuloplasty was reevaluated to enlarge the mitral annulus. For patients with a semirigid prosthetic ring, upsizing of the prosthetic ring by 1 or 2 sizes was performed. For patients with an autologous pericardial band, partial removal of the pericardial band was performed.

\section{Definition of Barlow Disease}

Barlow disease was defined when a patient possessed a valve with the following characteristics: a billowing valve with excess tissue and thickened leaflets; chords typically thickened and elongated; and a large valve with severe annular dilatation.

\section{Statistical Analysis}

Differences between groups were compared using the $\chi^{2}$ test for categorical variables and the Student $t$ test for continuous variables. To assess the effect of LV function on SAM, we calculated risk ratios (RR) and $95 \%$ confidence intervals $(\mathrm{CI})$ in contingency tables accompanied with the
Cochrane-Armitage trend test. Multivariable logistic regression analysis was performed to identify independent risk factors for development of SAM. Variables with a univariate $P$ value of less than .20 or those with known biological significance were included in the multivariable model. All statistical analyses were performed using SAS version 9.2 (SAS Institute, Inc, Cary, NC).

\section{RESULTS \\ Clinical Outcomes}

There was no operative mortality among the study patients. There were 27 patients ( 21 male, 6 female; mean age, $58.0 \pm 10.5$ years) in whom SAM developed (SAM cases), and 414 patients (280 male, 134 female; mean age, $55.2 \pm 14.5$ years) who had no SAM (noncases). The incidence of SAM was $6.1 \%$, and conservative management successfully resolved SAM in 15 of these patients. Revision of valve repair at the initial operation was performed in 12 patients, and SAM was resolved in 11; mitral valve replacement was required in the other patient. Among all 441 study patients, revision of repair at the initial operation was undertaken in $35(7.9 \%)$, and $34.3 \%$ of the revisions were performed as a result of SAM.

All SAM patients were discharged home without any symptom. According to the transthoracic echocardiogram at discharge, there was little or no residual MR in 23 (85.2\%) SAM patients and mild MR in $4(14.8 \%)$. SAM persisted at discharge in 4 patients; 3 patients exhibited mild MR associated with SAM and another patient was free of recurrent MR. In noncases, there was little or no residual MR in $380(91.8 \%)$ patients, mild MR in $32(7.7 \%)$, and moderate MR in $2(0.5 \%)$.

Preoperative patient characteristics, the location of mitral valve prolapse, and the operative procedures performed are summarized in Table 1. There were no differences between the 2 groups in preoperative patient characteristics, except for the prevalence of Barlow disease. The prevalence of SAM in patients with Barlow disease was $16.7 \%$ (Table E1). A solitary anterior lesion was rare in the SAM cases (1 patient), but the spectrum of valve lesions was not significantly different between the 2 groups. The performed operations were almost similar between the 2 groups. There were no differences in patient characteristics, location of mitral valve prolapse, and operative procedures between patients undergoing conservative treatment for SAM and those undergoing surgical treatment (Table E2).

\section{Influence of Preoperative LV Function on the Development of SAM}

The results of preoperative transthoracic echocardiography in the 2 groups are compared in Table 2. The SAM cases had significantly smaller systolic LV dimension (LVDs), thicker interventricular septum, and greater ejection fraction (EF). The incidence risks of SAM according to preoperative EF and LVDs, as well as RR with $95 \% \mathrm{CI}$ in each category to 
TABLE 1. Patient characteristics, location of mitral valve prolapse, and operative procedures

\begin{tabular}{|c|c|c|c|}
\hline & $\begin{array}{c}\text { SAM cases } \\
(\mathbf{n}=\mathbf{2 7})\end{array}$ & $\begin{array}{l}\text { Noncases } \\
(n=414)\end{array}$ & $\begin{array}{c}P \\
\text { value }\end{array}$ \\
\hline Age (y) & $58.0 \pm 10.5$ & $55.2 \pm 14.5$ & \\
\hline Male & $21(77.8 \%)$ & $280(67.6 \%)$ & \\
\hline NYHA class & $1.6 \pm 0.7$ & $1.9 \pm 0.8$ & \\
\hline Hypertension & $7(25.9 \%)$ & $122(29.5 \%)$ & \\
\hline Hyperlipidemia & $2(7.4 \%)$ & $41(9.9 \%)$ & \\
\hline Atrial fibrillation & $6(22.2 \%)$ & $125(30.2 \%)$ & \\
\hline \multicolumn{4}{|l|}{ Location of mitral valve prolapse } \\
\hline Anterior leaflet & $1(3.7 \%)$ & $78(18.8 \%)$ & \\
\hline Posterior leaflet & $19(70.4 \%)$ & $215(51.9 \%)$ & \\
\hline Both leaflets & $7(25.9 \%)$ & $121(29.2 \%)$ & \\
\hline Barlow disease & $7(25.9 \%)$ & $35(8.5 \%)$ & $<$. \\
\hline \multicolumn{4}{|l|}{ Operative procedures } \\
\hline Leaflet resection & $23(85.2 \%)$ & $338(81.6 \%)$ & \\
\hline With sliding technique & $11(40.7 \%)$ & $121(29.2 \%)$ & \\
\hline Chordal replacement & $14(51.9 \%)$ & $253(61.1 \%)$ & \\
\hline \multicolumn{4}{|l|}{ Types of artificial ring } \\
\hline Semirigid ring & $16(59.3 \%)$ & $300(72.5 \%)$ & \\
\hline Flexible ring & $11(40.7 \%)$ & $111(26.8 \%)$ & \\
\hline Tailor ring & 2 & 24 & \\
\hline Duran ring & 2 & 28 & \\
\hline Autologous pericardial band & 7 & 58 & \\
\hline Cosgrove band & 0 & 1 & \\
\hline None & 0 & $3(0.7 \%)$ & \\
\hline Ring size & $29.9 \pm 2.2$ & $29.7 \pm 3.0$ & \\
\hline \multicolumn{4}{|l|}{ Additional procedure } \\
\hline Maze & $4(14.8 \%)$ & $78(18.8 \%)$ & \\
\hline Tricuspid valve repair & $3(11.1 \%)$ & $53(12.8 \%)$ & 1.0 \\
\hline
\end{tabular}

Data are shown as mean \pm standard deviation or $\mathrm{n}(\%)$. SAM, Systolic anterior motion; NYHA, New York Heart Association.

a reference category, are shown in Table 3. No SAM occurred in patients with $\mathrm{LV}$ dysfunction $(\mathrm{EF}<60 \%)$. The incidence of SAM increased with greater preoperative EF $(P<.01$ for trend). Similarly, no SAM occurred in patients with an enlarged LV (LVDs $>46 \mathrm{~mm}$ ). The incidence of SAM increased as the preoperative LVDs became smaller $(P<.01$ for trend). The distribution of preoperative $\mathrm{EF}$ and LVDs in the 2 groups is shown in Figure 1. Most patients (26/27) in the SAM group exhibited well-preserved LV function $(\mathrm{EF} \geq 60 \%$ and LVDs $<40 \mathrm{~mm}$ ).

TABLE 2. Preoperative echocardiographic data

\begin{tabular}{lccc}
\hline & SAM cases $(\mathbf{n}=\mathbf{2 7})$ & Noncases $(\mathbf{n}=\mathbf{4 1 4})$ & $\boldsymbol{P}$ value \\
\hline LAD $(\mathrm{mm})$ & $44.6 \pm 8.2$ & $46.8 \pm 7.8$ & .16 \\
LVDd $(\mathrm{mm})$ & $54.9 \pm 5.6$ & $56.6 \pm 6.1$ & .17 \\
LVDs $(\mathrm{mm})$ & $32.0 \pm 5.4$ & $35.4 \pm 5.5$ & $<.01$ \\
IVS $(\mathrm{mm})$ & $10.8 \pm 1.5$ & $10.0 \pm 1.1$ & $<.01$ \\
LVPW $(\mathrm{mm})$ & $10.4 \pm 1.2$ & $10.0 \pm 1.1$ & .06 \\
EF $(\%)$ & $70.0 \pm 7.1$ & $65.1 \pm 6.9$ & $<.01$ \\
\hline
\end{tabular}

$P$ values set in italics indicate statistical significance. $S A M$, Systolic anterior motion; $L A D$, left atrial dimension; $L V D d$, diastolic left ventricular dimension; $L V D s$, systolic left ventricular dimension; IVS, interventricular septum; $L V P W$, left ventricular posterior wall; $E F$, ejection fraction.
TABLE 3. Incidence of SAM according to preoperative ejection fraction and left ventricular end-systolic dimension

\begin{tabular}{lrrrrr}
\hline & $\begin{array}{c}\text { SAM } \\
\text { Ejection fraction (\%) }\end{array}$ & $\begin{array}{c}\text { No. } \\
\text { at risk }\end{array}$ & Risk & RR* & $\mathbf{9 5 \%}$ CI \\
\hline $\begin{array}{l}\text { Ejection fraction (\%) } \\
\leq 49\end{array}$ & 0 & 9 & $0.0 \%$ & & \\
\hline $50-54$ & 0 & 16 & $0.0 \%$ & & \\
$55-59$ & 0 & 51 & $0.0 \%$ & & \\
$60-64 \dagger$ & 6 & 118 & $5.1 \%$ & 1.00 & Reference \\
$65-69$ & 8 & 134 & $6.0 \%$ & 1.17 & $0.42-3.29$ \\
$70-74$ & 7 & 81 & $8.6 \%$ & 1.70 & $0.59-4.87$ \\
$75+$ & 6 & 32 & $18.8 \%$ & 3.69 & $1.27-10.67$ \\
End-systolic & & & & & \\
$\quad$ diameter (mm) & & & & & \\
$\geq 46$ & 0 & 16 & $0.0 \%$ & & \\
$41-45$ & 1 & 47 & $2.1 \%$ & 0.52 & $0.06-4.19$ \\
$36-40 \dagger$ & 6 & 146 & $4.1 \%$ & 1.00 & Reference \\
$31-35$ & 10 & 159 & $6.3 \%$ & 1.53 & $0.57-4.11$ \\
$26-30$ & 7 & 58 & $12.1 \%$ & 2.94 & $1.03-8.37$ \\
$\leq 25$ & 3 & 15 & $20.0 \%$ & 4.87 & $1.35-17.5$ \\
\hline
\end{tabular}

SAM, Systolic anterior motion; $R R$, risk ratio; $C I$, confidence interval. *Risk ratio compared to reference category. $\dagger$ Reference category.

The incidence of SAM among the highest risk group (EF $\geq 75 \%$ and LVDs $\leq 25 \mathrm{~mm}$ ) was $37.5 \%$. The operative procedures performed for these patients were similar to those performed for the other patients, except for the more frequent use of sliding technique $(50.0 \%)$ and flexible ring $(62.5 \%)$. Patients with a small hyperkinetic heart received a smaller annular ring than the other patients $(28.8$ $\mathrm{mm}$ vs $29.8 \mathrm{~mm}$ ).

The results of multivariable analysis are shown in Table 4. A posterior leaflet lesion, Barlow disease, performance of the sliding leaflet technique, use of a semirigid ring, ring size, thickness of the interventricular septum, and $\mathrm{EF}$ were incorporated into the analysis. Interventricular septum thickness and EF were independent predictors for the development of SAM.

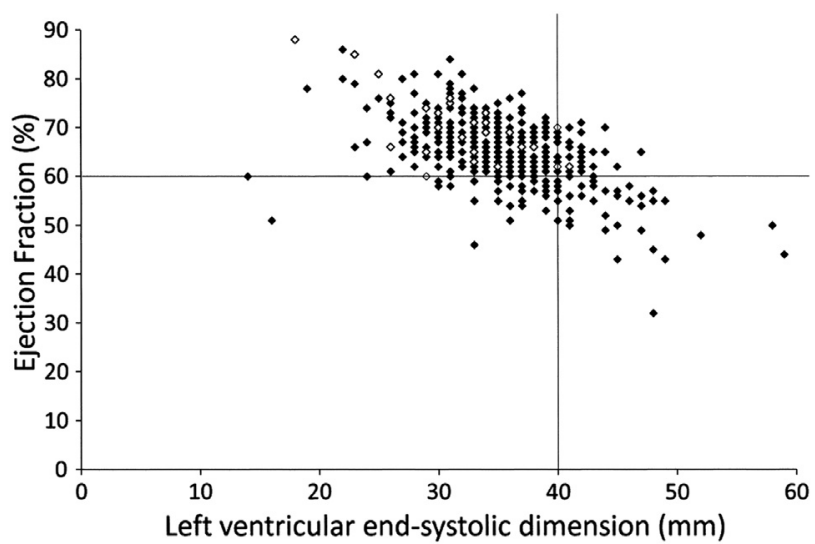

FIGURE 1. Distribution of preoperative ejection fractions and left ventricular dimensions in the 2 groups. White and black squares represent patients with and without systolic anterior motion, respectively. 
TABLE 4. Multivariable predictors of the development of SAM

\begin{tabular}{lccc}
\hline \multicolumn{1}{c}{ Variable } & Odds ratio & $\mathbf{9 5} \%$ CI & $\boldsymbol{P}$ value \\
\hline Posterior leaflet lesion & 4.77 & $0.59-38.8$ & .14 \\
Barlow's disease & 3.17 & $0.94-10.6$ & .06 \\
Sliding leaflet technique & 0.84 & $0.32-2.19$ & .72 \\
Semi rigid ring & 0.87 & $0.35-2.20$ & .77 \\
Ring size $(1 \mathrm{~mm})$ & 1.07 & $0.91-1.25$ & .42 \\
IVS $(1 \mathrm{~mm})$ & 1.60 & $1.15-2.21$ & $<.01$ \\
EF $(5 \%)$ & 1.66 & $1.19-2.31$ & $<.01$ \\
\hline
\end{tabular}

$P$ values set in italics indicate statistical significance. $S A M$, Systolic anterior motion; $C I$, confidence interval; IVS, interventricular septum; $E F$, ejection fraction.

Transthoracic echocardiography at discharge showed that SAM cases had smaller LVDs $(30.9 \pm 5.4 \mathrm{~mm}$ vs $34.7 \pm 6.5 \mathrm{~mm})$ and greater $\mathrm{EF}(58.0 \% \pm 10.5 \%$ vs $53 \% .5 \pm 9.6 \%$ ) than noncases.

\section{DISCUSSION}

The present study revealed a close relationship between the development of SAM and preoperative LV function in patients undergoing mitral valve repair. No SAM developed in patients with an impaired or enlarged LV. The incidence of SAM increased with LV hyperkinesia. In the group with the highest risk of SAM (EF $\geq 75 \%$ and LVDs $\leq 25 \mathrm{~mm}$ ), the incidence of SAM was as high as $37.5 \%$.

The contribution of LV function to the development of SAM has been discussed in several reports. Freeman and associates ${ }^{10}$ attributed SAM to the hyperdynamic state caused by post-cardiopulmonary bypass hypovolemia in combination with catecholamine infusion. Braun and coworkers ${ }^{2}$ reported that most SAM could be managed by conservative treatment consisting of volume expansion and discontinuation of inotropic drug therapy. Rescigno and colleagues ${ }^{4}$ reported a rare case of late development of SAM. In this patient, SAM emerged on recovery of LV function that had temporarily deteriorated immediately after surgery. These studies suggested that there was a relationship between LV function and the development of SAM, but the association was mainly based on the impression of the surgeons rather than on a systematic demonstration using objective data. Data analyzed in the present study demonstrated a close relationship between preoperative LV function and the incidence of SAM.

Although the present study revealed that preoperative LV function was associated with susceptibility to SAM, it did not deny a contribution of mitral valve structure or procedural modification to the development of SAM. Preoperative LV function was not the only determinant in the development of SAM. In the highest risk group (EF $\geq 75 \%$ and LVDs $\leq 25 \mathrm{~mm}$ ), about two thirds of patients did not have SAM, suggesting that the mechanism leading to SAM is multifactorial. The present study also suggested another possible risk factor for SAM. In patients with a solitary anterior lesion, the incidence of SAM was low, although the differences did not reach statistical significance. There were no differences in preoperative LV function between patients with a solitary anterior lesion and the other patients (LVDs, $36.3 \pm 5.6 \mathrm{~mm}$ vs $35.0 \pm 5.5 \mathrm{~mm}$; EF, $63.9 \% \pm 6.5 \%$ vs $65.7 \% \pm 7.1 \%$ ). Patients with a solitary anterior lesion underwent posterior leaflet resection less frequently ( $0 \%$ vs $93.6 \%)$. Posterior leaflet resection might immobilize the posterior leaflet motion and fix it at the closed position, which might bring the coaptation point closer to the LV outflow tract and predispose the mitral valve toward developing SAM. Patients with Barlow disease also exhibited a high risk of SAM, although the difference did not reach statistical significance in multivariable analysis. However, our study did not include the measurement of the leaflet length, which made it difficult to precisely analyze the relationship between leaflet length and the risk of SAM.

The findings of the present study may attain further importance in the modern era of mitral valve surgery. According to the current American College of Cardiology/American Heart Association guidelines, surgical correction is recommended for patients with severe mitral regurgitation before the emergence of early signs of LV deterioration. ${ }^{11}$ Several studies demonstrated superior results with "early surgery" compared with "conventional treatment."12,13 Therefore, increasing numbers of patients with well-preserved LV function are now undergoing mitral valve surgery. According to our results, the risk of SAM was only $0.96 \%$ (1/104) in patients with LV dysfunction and who are recommended to receive a "conventional treatment" strategy. However, the risk of SAM was 7.7\% (26/337) in "early surgery" patients. Therefore, surgeons in the modern era of mitral valve surgery must be skilled in managing this complication.

The present study revealed that a small hyperkinetic heart is a risk factor for SAM. Considering the high incidence of SAM, a small hyperkinetic heart should be treated with caution. We routinely administered catecholamine at bypass discontinuation, which may have contributed to the development of SAM and should be avoided in patients with a hyperkinetic heart. In this study, patients with a small hyperkinetic heart received a smaller annular ring than the other patients. At the revision of the repair, we upsized the prosthetic ring by 1 or 2 sizes to eliminate SAM. A ring size is considered the culprit in the development of SAM, and a small ring should be avoided for patients with a small hyperkinetic heart. Other preventive procedures ${ }^{14,15}$ may also be required, not only in patients with a high posterior leaflet, but also in patients with a hyperkinetic LV.

\section{Study Limitations}

This study has several limitations. First, all data were retrospectively collected; therefore, we cannot eliminate the possibility of confounding bias in the interpretation of the data. We carried out procedures to correct for morphologic characteristics that may predispose some mitral valves to 
the development of SAM. Therefore, we could not assess the contribution of these characteristics. We could not incorporate some variables into multivariate analysis, including aorta-mitral angle, the length of the anterior leaflet, and the existence of a sigmoid septum, which might have an influence on the susceptibility to the development of SAM. Some data on cardiac chamber parameters in this study may seem small compared with those from Western countries, but this is likely related to the small physiques of the Japanese compared with those of Western populations. Such differences in physique must be considered in the interpretation of our data.

\section{References}

1. Crescenzi G, Landoni G, Zangrillo A, Guarracino F, Rosica C, La Canna G, et al. Management and decision-making strategy for systolic anterior motion after mitral valve repair. J Thorac Cardiovasc Surg. 2009;137:320-5.

2. Braun ML, Abel MD, Click RL, Morford RG, Dearani JA, Sundt TM, et al. Systolic anterior motion after mitral valve repair: is surgical intervention necessary? J Thorac Cardiovasc Surg. 2007;133:136-43.

3. Zegdi R, Carpentier A, Doguet F, Berrebi A, Khabbaz Z, Chauvaud S, et al. Systolic anterior motion after mitral valve repair: an exceptional cause of late failure. J Thorac Cardiovasc Surg. 2005;130:1453-4.

4. Rescigno G, Cecconi M, Matteucci MLS, Domenella P, Munch C, Iacobone G. Delayed systolic anterior motion after mitral valve repair. $J$ Heart Valve Dis. 2006;15:512-4.

5. Carpentier A, Adams DH, Filsoufi F. Techniques in systolic anterior motion (SAM). In: Carpentier A, Adams DH, Filsoufi F, eds. Carpentier's reconstructive valve surgery. Philadelphia: Saunders, Elsevier; 2010. 157-65.

6. Grossi EA, Galloway AC, Parish MA, Asai T, Gindea AJ, Harty S, et al. Experience with twenty-eight cases of systolic anterior motion after mitral valve reconstruction by the Carpentier technique. J Thorac Cardiovasc Surg. 1992; 103:466-70.

7. Maslow AD, Regan MM, Hearing M, Johnson RG, Levine RA. Echocardiographic predictors of left ventricular outflow tract obstruction and systolic anterior motion of the mitral valve after mitral valve reconstruction for myxomatous valve disease. J Am Coll Cardiol. 1999;34:2096-104.

8. Lee KS, Stewart WJ, Lever HM, Underwood PL, Cosgrove DM. Mechanism of outflow tract obstruction causing failed mitral valve repair. Anterior displacement of leaflet coaptation. Circulation. 1993;88(5 Pt 2):II24-9.

9. Shah PM, Raney AA. Echocardiographic correlates of left ventricular outflow obstruction and systolic anterior motion following mitral valve repair. $J$ Heart Valve Dis. 2001;10:302-6.

10. Freeman WK, Schaff HV, Khandheria BK, Oh JK, Orszulak TA, Abel MD, et al Intraoperative evaluation of mitral valve regurgitation and repair by transesophageal echocardiography: incidence and significance of systolic anterior motion. J Am Coll Cardiol. 1992;20:599-609.

11. Bonow RO, Carabello BA, Chatterjee K, de Leon AC, Faxon DP, Freed MD, et al ACC/AHA 2006 guidelines for the management of patients with valvular heart disease: a report of the American College of Cardiology/American Heart Association Task Force on Practice Guidelines (writing Committee to Revise the 1998 guidelines for the management of patients with valvular heart disease). J Am Coll Cardiol. 2006;48:e1-148.

12. Montant P, Chenot F, Robert A, Vancraeynest D, Pasquet A, Gerber B, et al Long-term survival in asymptomatic patients with severe degenerative mitral regurgitation: a propensity score-based comparison between an early surgical strategy and a conservative treatment approach. J Thorac Cardiovasc Surg. 2009;138:1339-48

13. Knag DH, Kim JH, Rim JH, Kim MJ, Yun SC, Song JM, et al. Comparison of early surgery versus conventional treatment in asymptomatic severe mitral regurgitation. Circulation. 2009;119:797-804.

14. Asai T, Kinoshita T, Hosoba S, Takashima N, Kambara A, Suzuki T, et al. Butterfly resection is safe and avoids systolic anterior motion in posterior leaflet prolapse repair. Ann Thorac Surg. 2011;92:2097-103.

15. Quigley RL. Prevention of systolic anterior motion after repair of the severely myxomatous mitral valve with an anterior leaflet valvuloplasty. Ann Thorac Surg. 2005;80:179-82. 
TABLE E1. Incidence of SAM according to patient and operative characteristics

\begin{tabular}{lcc}
\hline & \multicolumn{2}{c}{ Incidence of SAM } \\
\cline { 2 - 3 } \multicolumn{1}{c}{ Characteristics } & With characteristics & Without characteristics \\
\hline Posterior lesion & $7.2 \%$ & $1.3 \%$ \\
Barlow disease & $16.7 \%$ & $5.0 \%$ \\
Sliding leaflet resection & $8.3 \%$ & $5.2 \%$ \\
Chordal replacement & $5.2 \%$ & $7.5 \%$ \\
Semirigid ring & $5.1 \%$ & $8.8 \%$ \\
\hline
\end{tabular}

SAM, Systolic anterior motion.
TABLE E2. Comparisons between conservative and surgical treatment in SAM cases

\begin{tabular}{|c|c|c|c|}
\hline & $\begin{array}{c}\text { Conservative } \\
\text { treatment } \\
(\mathbf{n}=\mathbf{1 5}) \\
\end{array}$ & $\begin{array}{c}\text { Surgical } \\
\text { treatment } \\
(n=12) \\
\end{array}$ & $\begin{array}{c}P \\
\text { value } \\
\end{array}$ \\
\hline Age $(y)$ & $57.5 \pm 9.3$ & $58.7 \pm 12.2$ & .78 \\
\hline Male & $12(80.0 \%)$ & $9(75.0 \%)$ & 1.00 \\
\hline \multicolumn{4}{|l|}{$\begin{array}{l}\text { Location of mitral valve } \\
\text { prolapse }\end{array}$} \\
\hline Anterior leaflet & $1(6.7 \%)$ & $0(0 \%)$ & .10 \\
\hline Posterior leaflet & $10(66.7 \%)$ & $9(75.0 \%)$ & \\
\hline Both leaflets & $4(26.7 \%)$ & $3(25.0 \%)$ & \\
\hline Barlow disease & $3(20.0 \%)$ & $4(33.3 \%)$ & .66 \\
\hline \multicolumn{4}{|l|}{ Operative procedures } \\
\hline Leaflet resection & $11(73.3 \%)$ & $12(100 \%)$ & .11 \\
\hline With sliding technique & $8(53.3 \%)$ & $5(41.7 \%)$ & \\
\hline Chordal replacement & $6(40.0 \%)$ & $6(50.0 \%)$ & 1.00 \\
\hline Types of artificial ring & & & 1.00 \\
\hline Semirigid ring & $9(60.0 \%)$ & $7(58.3 \%)$ & \\
\hline Flexible ring & $6(40.0 \%)$ & $5(41.7 \%)$ & \\
\hline Ring size & $30.1 \pm 2.1$ & $29.8 \pm 2.3$ & .72 \\
\hline $\mathrm{LAD}(\mathrm{mm})$ & $43.5 \pm 8.0$ & $46.1 \pm 8.6$ & .43 \\
\hline $\operatorname{LVDd}(\mathrm{mm})$ & $54.7 \pm 4.7$ & $55.2 \pm 6.7$ & .83 \\
\hline LVDs (mm) & $32.5 \pm 4.0$ & $31.4 \pm 7.0$ & .63 \\
\hline IVS (mm) & $10.7 \pm 1.8$ & $10.8 \pm 1.2$ & .94 \\
\hline LVPW (mm) & $10.3 \pm 1.2$ & $10.6 \pm 1.1$ & .52 \\
\hline $\mathrm{EF}(\%)$ & $67.9 \pm 4.8$ & $72.6 \pm 8.7$ & .11 \\
\hline
\end{tabular}

